\title{
EP-198
}

\section{HDAC-targeting epigenetic drug screening for biliary tract cancer}

\author{
Christian MAYR ${ }^{1,2}$, Tobias KIESSLICH ${ }^{1,2}$, Sara ERBER ${ }^{1}$, Dino BEKRIC ${ }^{1}$, Heidemarie DOBIAS ${ }^{1}$, Markus RITTER ${ }^{1,3}$ \\ Tarkan JÄGER ${ }^{4}$, Bettina NEUMAYER ${ }^{5,6}$, Paul WINKELMANN ${ }^{5,6}$, Eckhard KLIESER ${ }^{5,6}$, Daniel NEUREITER ${ }^{* 5,6}$ \\ ${ }^{1}$ Institute for Physiology and Pathophysiology, Paracelsus Medical University, Salzburg, Austria \\ 2Department of Internal Medicine I, University Clinics Salzburg, Paracelsus Medical University, Salzburg, Austria \\ ${ }^{3}$ Ludwig Boltzmann Institute for Arthritis und Rehabilitation, Paracelsus Medical University, Salzburg, Austria \\ ${ }^{4}$ Department of Surgery, University Clinics Salzburg, Paracelsus Medical University, Salzburg, Austria \\ ${ }^{5}$ Institute of Pathology, University Clinics Salzburg, Paracelsus Medical University, Salzburg, Austria \\ ${ }^{6}$ Institute of Pathology, Cancer Cluster Salzburg, Salzburg, Austria
}

Introduction: Although molecular insights about biliary tract cancer (BTC) increased in the last decade, new therapeutic strategy like inhibition of histone deacetylases (HDACs) could additionally improve the still dismal outcome of this tumor entity.

Methods: Therefore, we performed comprehensive investigation of HDAC expression and pharmacological inhibition in a panel of eight established BTC cell lines and in a cohort resected native BTC specimens $(\mathrm{n}=78)$.

Results: HDAC profiling revealed a heterogeneous expression of HDACs across the studied cell lines and the BTC cancer specimen. Cytotoxicity of six established HDAC inhibitors (HDACi) covering pan- and class-specific HDACis was dose- as well as cell line-dependent and did not show a statistical correlation with HDAC isoform expression. Romidepsin (a class II HDACi), induced the highest reduction of cell viability and apoptosis in BTC cells which was paralleled by reducing HDAC1/2 activity and increasing histone 3 lysine 9 acetylation. Furthermore, non-toxic concentrations of romidepsin could augment the cytotoxic effect of the standard chemotherapeutic cisplatin. Related to the clinical tumor specimen, HDAC expression pattern correlated with the tumor grading and the survival of BTC patients.

Conclusions: In conclusion, in-vitro-experiments provide clear evidence that the HDAC class I inhibitor romidepsin is effective for BTC alone and acts supportively in combination with standard chemotherapeutics. Additionally, the observed HDAC expression in BTC specimens could serve as a predictive and prognostic biomarker for BTC patients. 